\section{Kompass \\ Ophthalmologie}

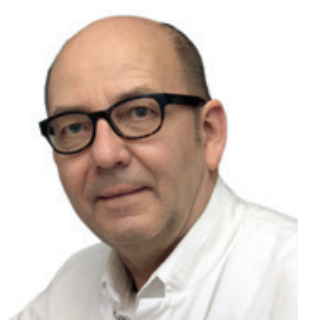

\section{Uwe Pleyer}

Klinik für Augenheilkunde, Charité -

Universitätsmedizin Berlin, Campus Virchow Klinikum, Berlin, Deutschland

Verehrte Kolleginnen und Kollegen,

willkommen zu einer neuen Ausgabe von Kompass Ophthalmologie.

Sie betreffen ca. 5-10\% der Weltbevölkerung - Tendenz steigend - und bilden nach den Herz-Kreislauf-Erkrankungen und Neoplasien die dritthäufigste Erkrankungsgruppe: Die Rede ist von Autoimmunerkrankungen [1]. Als Schwerpunkt dieses Heftes haben wir dieses umfassende Thema gewählt und stellen «Autoimmunität und Auge» in den Fokus dieser Ausgabe von Kompass Ophthalmologie.

Im öffentlichen Bewusstsein und auch bei vielen ärztlichen Kollegen werden mit Autoimmunerkrankungen vor allem rheumatologische Erkrankungen, der Diabetes mellitus (Typ I) oder auch die Multiple Sklerose assoziiert. Autoimmunerkrankungen des Auges werden demgegenüber weniger wahrgenommen und in ihrer Bedeutung deutlich «unterschätzt». Nahezu alle Strukturen des Auges können davon betroffen sein. Ganz im Vordergrund stehen dabei intraokulare Entzündungen. Da sie häufig chronisch und rezidivierend verlaufen, sind die, überwiegend jungen, Patienten oft schwer betroffen und langfristig in ihrer Lebensqualität eingeschränkt. Wie bei allen Autoimmunerkrankungen wird für die Pathogenese intraokularer Entzündungen ein Toleranzverlust ge-

\title{
Autoimmunität und Auge
}

genüber körpereigenen Gewebestrukturen, z.B. retinalen Antigenen, angenommen. Für die vorliegende Ausgabe von Kompass Ophthalmologie haben wir 3 Arbeiten ausgewählt, die unterschiedliche Aspekte der intraokularen Entzündung aufgreifen.

Frau Dingerkus und Herr Becker (Zürich, CH) stellen in der Rubrik «Transfer in die Praxis» eine kürzlich in «Ophthalmology» erschienene Publikation vor, die sich der Frage prädiktiver Faktoren für Remission und Verlauf bei anteriorer Uveitis widmen. Die Orginalarbeit kann dabei auf einen sehr großen $\mathrm{Pa}$ tientenpool mit nahezu 3000 Augen zurückgreifen und erlaubt damit valide Aussagen zu wichtigen, klinischen Aspekten. So berichten die Autoren, dass Rezidive intraokularer Entzündungen signifikant mit jungem Lebensalter und beidseitiger Uveitis assoziiert sind. Besonders schwer betroffen und damit Zielgruppe einer intensiven immunmodulatorischen Behandlung sind Kinder, die von einer juvenilen Arthritis betroffen sind. Interessant, dass andererseits das HLA-B 27 Antigen keinen wesentlichen Einfluss auf die Remissionsrate aufwies. Sind Sie neugierig geworden? Weitere spannende Ergebnisse dieser Arbeit finden sie auf Seite 127 in der aktuellen Ausgabe von Kompass Ophthalmologie.

Einen Durchbruch in der Behandlung von Autoimmunerkrankungen stellte die Ein- führung von «Biologika» dar. Es handelt sich dabei im Wesentlichen um spezifische monoklonale Antikörper, die gezielt Mediatoren des Immunsystems supprimieren. Als sehr effektiv haben sich dabei Inhibitoren von Tumornekrosefaktor-alpha (TNFi) erwiesen. Inzwischen ist die Behandlung mit TNFi seit einigen Jahren bei vielen Autoimmunerkrankungen etabliert. Doch wie steht es um unerwünschte Wirkungen? Frau Fernanda N. Susanna und Carlos Pavesio (São Paulo, Brasilien und London, UK) haben diese Frage aufgegriffen und die aktuelle Literatur zu okulären Nebenwirkungen von TNFi systematisch untersucht. Sie stellen dabei heraus, dass im Vordergrund unerwünschter Wirkungen paradoxe Reaktionen auftraten, d.h. unter der Behandlung mit TNFi sind erstmals Entzündungen aufgetreten. Dieses Phänomen wird immer wieder beobachtet und kann sich an unterschiedlichen Organsystemen zeigen. Das Phänomen wird mit einer gestörten Zytokin-Homöostase TNF vs. Interferon erklärt. Welche TNFi besonders häufig zu paradoxen Wirkungen am Auge führen und welche Konsequenzen dies für Ihre Patienten bedeuten kann, können Sie auf Seite 120 in diesem Heft nachlesen.

Abschließend möchte ich noch auf eine interessante Kasuistik hinweisen (Ann Arbor, USA). Sie ist bereits im Titel mit «Autoim- information@karger.com @ (c) 2020 S. Karger GmbH, Freiburg www.karger.com/kop

\section{Karger"'}

Prof. Dr. Uwe Pleyer

Klinik für Augenheilkunde

Charité - Universitätsmedizin Berlin, Campus Virchow Klinikum

Augustenburger Platz 1, 13353 Berlin, Deutschland

uwe.pleyer@charite.de 
mun-Retinopathie» überschrieben und berichtet von einer bisher selten beobachteten Assoziation. Autoimmun-Retinopathien können unter anderem mit malignen Erkrankungen (Karzinomen u.a.) im Zusammenhang stehen. Bei der hier vorgestellten Patientin lagen typische klinische Veränderungen mit Visusminderung, Skotomen, Nachtblindheit und Photopsien vor. Bei weiterer Abklärung stellte sich eine monoklonale Gammopathie unklarer Signifikanz, kurz MGUS, heraus. Diese Erkrankung ist keinesfalls selten und tritt oft asymptomatisch als prämaligne Proliferation von Plasmazellen auf. Bei der hier vorgestellten Patientin wurden Antikörper gegen AlphaEnolase und Carbonanhydrase II nachgewiesen, die von den Autoren als autoimmunologische Auslöser angesehen wer- den. Wie die Autoren bei dieser Patientin auf die richtige Spur gelenkt wurden und welche Maßnahmen zur erfolgreichen Behandlung eingesetzt wurden, können Sie auf Seite 155 weiterverfolgen.

Aus den vorgestellten Beiträgen können wir resümieren, dass ein weites Spektrum autoimmunologischer Veränderungen am Auge auftreten kann. Die richtige Einordnung klinischer Leitbefunde, eine gezielte Anamnese und ein differenzialdiagnostischer Spürsinn sind wichtig, um zügig zu einer Diagnose zu gelangen. Die Behandlungsmöglichkeiten haben sich zwar deutlich verbessert, dennoch sind vergleichsweise wenige Wirkstoffe gezielt für Autoimmunerkrankungen des Auges geprüft und zugelassen. Daher ist ein stärkeres Engagement notwendig, um eine bessere Versor- gung unserer Patienten zu gewährleisten. Es bleibt daher ein in jeder Hinsicht spannendes Thema mit hoher Dynamik. Dies geht nicht zuletzt auch aus den vorgestellten Artikeln hervor.

Wir freuen uns über Ihr Interesse und wünschen Ihnen Lesevergnügen.

Bleiben Sie gesund und aufmerksam! Herzlichst,

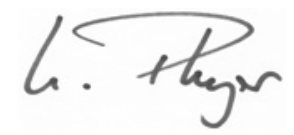

Ihr

Uwe Pleyer

und das Team von Kompass Ophthalmologie

\section{Literatur}

1 Deutsche Autoimmun-Stiftung: Erkrankungen. www.autoimmun.org/erkrankungen 